\title{
Multiple Positive Solutions of $m$-Point BVPs for Third-Order $p$-Laplacian Dynamic Equations on Time Scales
}

\author{
Li-Hua Bian, ${ }^{1}$ Xi-Ping He, ${ }^{1,2}$ and Hong-Rui Sun ${ }^{1}$ \\ ${ }^{1}$ School of Mathematics and Statistics, Lanzhou University, Lanzhou, Gansu 730000, China \\ ${ }^{2}$ Center of Teaching Guidance, Gansu Radio and TV University, Lanzhou, Gansu 730000, China \\ Correspondence should be addressed to Hong-Rui Sun, hrsun@lzu.edu.cn
}

Received 17 August 2009; Accepted 26 October 2009

Recommended by Alberto Cabada

This paper is concerned with the existence of multiple positive solutions for the third-order $p$ Laplacian dynamic equation $\left(\phi_{p}\left(u^{\Delta \nabla}(t)\right)\right)^{\nabla}+a(t) f\left(t, u(t), u^{\Delta}(t)\right)=0, t \in[0, T]_{\mathbb{T}}$ with the multipoint boundary conditions $u^{\Delta}(0)=u^{\Delta \nabla}(0)=0, u(T)+B_{0}\left(\sum_{i=1}^{m-2} b_{i} u^{\Delta}\left(\xi_{i}\right)\right)=0$, where $\phi_{p}(u)=|u|^{p-2} u$ with $p>1$. Using the fixed point theorem due to Avery and Peterson, we establish the existence criteria of at least three positive solutions to the problem. As an application, an example is given to illustrate the result. The interesting points are that not only do we consider third-order $p$-Laplacian dynamic equation but also the nonlinear term $f$ is involved with the first-order delta derivative of the unknown function.

Copyright (C) 2009 Li-Hua Bian et al. This is an open access article distributed under the Creative Commons Attribution License, which permits unrestricted use, distribution, and reproduction in any medium, provided the original work is properly cited.

\section{Introduction}

The theory of dynamic equations on time scales was introduced by Stefan Hilger in 1988 [1]. This theory has attracted many researchers' attention and interest since it cannot only unify differential and difference equations but also provides accurate information of phenomena that manifest themselves partly in continuous time and partly in discrete time. In addition, time-scale calculus would allow exploration of a variety of situations in economic, biological, heat transfer, stock market, and epidemic models [2,3], and so forth.

Recently, there has been much attention paid to the existence of positive solutions for second-order nonlinear boundary value problems on time scales; see [4-10] and the references therein. On the one hand, higher-order nonlinear boundary value problems have been studied extensively; see [11-14] and the references therein. On the other hand, the boundary value problems with $p$-Laplacian operator have also been discussed extensively 
in literature; for example, see [15-17]. However, very little work has been done to the thirdorder $p$-Laplacian dynamic equations on time scales $[18,19]$.

For convenience, throughout this paper, we denote $\phi_{p}(s)$ as $p$-Laplacian operator, that is, $\phi_{p}(s)=|s|^{p-2} s$ for $p>1$ with $\phi_{p}^{-1}=\phi_{q}$ and $1 / p+1 / q=1$. We also assume that $\mathbb{T}$ is a closed subset of $\mathbb{R}$ with $0, T \in \mathbb{T}$; an interval $(0, T)_{\mathbb{T}}$ always means $(0, T) \cap \mathbb{T}$. Other types of intervals are defined similarly.

For example, Sun and Li [16] studied the two-point boundary value problem:

$$
\begin{gathered}
\left(\phi_{p}\left(u^{\Delta}(t)\right)\right)^{\Delta}+h(t) f\left(u^{\sigma}(t)\right)=0, \quad t \in[a, b]_{\mathbb{T}}, \\
u(a)-B_{0}\left(u^{\Delta}(a)\right)=0, \quad u^{\Delta}(\sigma(b))=0 .
\end{gathered}
$$

They established the existence theory for positive solutions by using various fixed point theorems [20, 21].

In [15], Su et al. investigated the existence of positive solutions for the following singular $p$-Laplacian $m$-point boundary value problem on time scales:

$$
\begin{gathered}
\left(\phi_{p}\left(u^{\Delta}(t)\right)\right)^{\nabla}+a_{1}(t) f(u(t))=0, \quad t \in(0, T)_{\mathbb{T}}, \\
u(0)=0, \quad u(T)-\sum_{i=1}^{m-2} \psi_{i}\left(u\left(\xi_{i}\right)\right)=0 .
\end{gathered}
$$

The main techniques are Schauder fixed point theorem and upper and lower solutions method.

In [19], Han and Kang considered the following third-order $p$-Laplacian dynamic equation on time scales:

$$
\begin{gathered}
\left(\phi_{p}\left(u^{\Delta \Delta}(t)\right)\right)^{\nabla}+f(t, u(t))=0, \quad t \in[a, b], \\
\alpha u(\rho(a))-\beta u^{\Delta}(\rho(a))=0, \quad \gamma u(b)+\delta u^{\Delta}(b)=0, \quad u^{\Delta \Delta}(\rho(a))=0 .
\end{gathered}
$$

By using fixed point theorems in cones, the existence criteria of multiple positive solutions are established.

In [10], Zhao and Sun studied the following second-order nonlinear three-point boundary value problem on time scales:

$$
\begin{gathered}
u^{\Delta \nabla}(t)+q(t) f\left(t, u(t), u^{\Delta}(t)\right)=0, \quad t \in(0, T)_{\mathbb{T}}, \\
\beta u(0)-\gamma u^{\Delta}(0)=0, \quad \alpha u(\eta)=u(T) .
\end{gathered}
$$

They gave sufficient condition for the existence of three positive solutions by using a fixed point theorem due to Avery and Peterson [22]. 
Motivated by $[10,15,16,19]$, in this paper we consider the following third-order $p$ Laplacian dynamic equation on time scales:

$$
\left(\phi_{p}\left(u^{\Delta \nabla}(t)\right)\right)^{\nabla}+a(t) f\left(t, u(t), u^{\Delta}(t)\right)=0, \quad t \in[0, T]_{\mathbb{T}}
$$

subject to the boundary condition

$$
u^{\Delta}(0)=u^{\Delta \nabla}(0)=0, \quad u(T)+B_{0}\left(\sum_{i=1}^{m-2} b_{i} u^{\Delta}\left(\xi_{i}\right)\right)=0
$$

where $0<\xi_{1}<\xi_{2}<\cdots<\xi_{m-2}<\rho(T), b_{i} \in[0, \infty)$ for $i=1,2, \ldots, m-2$. By using fixed point theorem due to Avery and Peterson [22], we prove that the boundary value problems (1.5) and (1.6) have at least three positive solutions under suitable assumptions. The interesting points are that not only do we consider third-order $p$-Laplacian dynamic equation on time scales but also the nonlinear term $f$ is involved with the first-order delta derivative of the unknown function.

Throughout this paper, it is assumed that

(H1) $a \in C_{l d}\left([0, T]_{\mathbb{T}}, \mathbb{R}^{+}\right)$and $f \in C\left([0, T]_{\mathbb{T}} \times \mathbb{R}^{+} \times \mathbb{R}, \mathbb{R}^{+}\right)$, both $a$ and $f$ do not vanish identically on any closed subinterval of $[0, T]_{\mathbb{T}}$, and there exists $l \in\left(0, \xi_{1}\right]_{\mathbb{T}}$ such that $\int_{0}^{l} a(\tau) \nabla \tau>0$ hold;

(H2) there exist nonnegative constants $B_{1}$ and $B_{2}$ satisfying $B_{1} x \leq B_{0}(x) \leq B_{2} x$ for $x \in \mathbb{R}$.

\section{Preliminary}

To prove the main results in this paper, we will employ several lemmas. And the following lemma is based on the linear BVP:

$$
\begin{gathered}
\left(\phi_{p}\left(u^{\Delta \nabla}(t)\right)\right)^{\nabla}+h(t)=0, \quad t \in[0, T]_{\mathbb{T}} \\
u^{\Delta}(0)=u^{\Delta \nabla}(0)=0, \quad u(T)+B_{0}\left(\sum_{i=1}^{m-2} b_{i} u^{\Delta}\left(\xi_{i}\right)\right)=0 .
\end{gathered}
$$

Lemma 2.1. If $h \in C_{l d}\left([0, T]_{\mathbb{T}}, \mathbb{R}^{+}\right)$, then the problems (2.1) and (2.2) have the unique nonnegative solution:

$$
\begin{aligned}
u(t)= & \int_{0}^{t}(t-s) \phi_{q}\left(-\int_{0}^{s} h(\tau) \nabla \tau\right) \nabla s+\int_{0}^{T}(T-s) \phi_{q}\left(\int_{0}^{s} h(\tau) \nabla \tau\right) \nabla s \\
& -B_{0}\left(\sum_{i=1}^{m-2} b_{i} \int_{0}^{\xi_{i}} \phi_{q}\left(\int_{0}^{s}-h(\tau) \nabla \tau\right) \nabla s\right) .
\end{aligned}
$$


Proof. For any $h \in C_{l d}\left([0, T]_{\mathbb{T}}, \mathbb{R}^{+}\right)$, suppose that $u$ is a solution of the BVPs (2.1) and (2.2). By integrating (2.1) from 0 to $t$, and combining the boundary condition, it follows that

$$
\begin{gathered}
u^{\Delta \nabla}(t)=\phi_{q}\left(-\int_{0}^{t} h(s) \nabla s\right), \quad u^{\Delta}(t)=\int_{0}^{t} \phi_{q}\left(-\int_{0}^{s} h(\tau) \nabla \tau\right) \nabla s, \\
u(t)-u(0)=\int_{0}^{t}\left(\int_{0}^{s} \phi_{q}\left(-\int_{0}^{\tau} h(r) \nabla r\right) \nabla \tau\right) \Delta s=\int_{0}^{t}(t-s) \phi_{q}\left(-\int_{0}^{s} h(\tau) \nabla \tau\right) \nabla s .
\end{gathered}
$$

Using (2.2), we can easily obtain

$$
u(0)=\int_{0}^{T}(T-s) \phi_{q}\left(\int_{0}^{s} h(\tau) \nabla \tau\right) \nabla s-B_{0}\left(\sum_{i=1}^{m-2} b_{i} \int_{0}^{\xi_{i}} \phi_{q}\left(\int_{0}^{s}-h(\tau) \nabla \tau\right) \nabla s\right)
$$

So

$$
\begin{aligned}
u(t)= & \int_{0}^{t}(t-s) \phi_{q}\left(-\int_{0}^{s} h(\tau) \nabla \tau\right) \nabla s+\int_{0}^{T}(T-s) \phi_{q}\left(\int_{0}^{s} h(\tau) \nabla \tau\right) \nabla s \\
& -B_{0}\left(\sum_{i=1}^{m-2} b_{i} \int_{0}^{\xi_{i}} \phi_{q}\left(\int_{0}^{s}-h(\tau) \nabla \tau\right) \nabla s\right) .
\end{aligned}
$$

Then it is easy to see that

$$
\begin{gathered}
\int_{0}^{t}(t-s) \phi_{q}\left(-\int_{0}^{s} h(\tau) \nabla \tau\right) \nabla s+\int_{0}^{T}(T-s) \phi_{q}\left(\int_{0}^{s} h(\tau) \nabla \tau\right) \nabla s \geq 0, \\
-B_{0}\left(\sum_{i=1}^{m-2} b_{i} \int_{0}^{\xi_{i}} \phi_{q}\left(\int_{0}^{s}-h(\tau) \nabla \tau\right) \Delta s\right) \geq B_{2} \sum_{i=1}^{m-2} b_{i} \int_{0}^{\xi_{i}} \phi_{q}\left(\int_{0}^{s} h(\tau) \nabla \tau\right) \Delta s \geq 0 .
\end{gathered}
$$

So $u(t) \geq 0$. On the other hand, it is easy to verify that if $u$ is as in (2.3), then $u$ is a solution of (2.1) and (2.2). Thus $u$ in (2.3) is the unique solution of (2.1) and (2.2).

Let $X=C_{l d}^{\Delta}[0, T]_{\mathbb{T}}$ be endowed with the norm

$$
\|u\|_{1}=\max \left\{\sup _{t \in[0, T]_{\mathbb{T}}}|u(t)| \sup _{t \in[0, T]_{\mathbb{T}^{\kappa}}}\left|u^{\Delta}(t)\right|\right\}, \quad u \in X .
$$

It follows that $\left(X,\|\cdot\|_{1}\right)$ is a Banach space. Define the cone $P \subset X$ by

$$
P=\left\{u \in X \mid u(t) \geq 0, u^{\Delta}(0) \leq 0, u(T)+B_{0}\left(\sum_{i=1}^{m-2} b_{i} u^{\Delta}\left(\xi_{i}\right)\right) \leq 0, u^{\Delta \nabla}(t) \leq 0, t \in(0, T)_{\mathbb{T}}\right\}
$$


Lemma 2.2. If $u \in P$, then there exists a constant $K$ such that

$$
\sup _{t \in[0, T]_{\mathbb{T}}}|u(t)| \leq K \sup _{t \in[0, T]_{\mathbb{T}^{k}}}\left|u^{\Delta}(t)\right|
$$

Proof. For $u \in P, u^{\Delta \nabla}(t) \leq 0$ implies that

$$
u(t)-u(T) \leq u^{\Delta}(T)(t-T) \leq T \sup _{t \in[0, T]_{\mathbb{T}}}\left|u^{\Delta}(t)\right|
$$

In addition, since

$$
u(T) \leq-B_{0}\left(\sum_{i=1}^{m-2} b_{i} u^{\Delta}\left(\xi_{i}\right)\right) \leq-B_{1} \sum_{i=1}^{m-2} b_{i} u^{\Delta}\left(\xi_{i}\right) \leq B_{1} \sum_{i=1}^{m-2} b_{i} \sup _{t \in[0, T]_{\mathbb{T}}}\left|u^{\Delta}(t)\right|
$$

then we have

$$
\sup _{t \in[0, T]_{T}}|u(t)| \leq\left(T+B_{1} \sum_{i=1}^{m-2} b_{i}\right) \sup _{t \in[0, T]_{T}}\left|u^{\Delta}(t)\right|
$$

Therefore, We can choose $K=T+B_{1} \sum_{i=1}^{m-2} b_{i}$ and the proof is complete.

Lemma 2.3. If $u \in P$, then $u(t) \geq((T-t) / T) \sup _{t \in[0, T]_{\mathbb{T}}}|u(t)|$ for $t \in[0, T]_{\mathbb{T}}$.

Proof. If $u \in P$, then $u^{\Delta}(t)$ is decreasing and $u^{\Delta}(0) \leq 0$, and thus $u^{\Delta}(t) \leq 0$ and $u(t)$ are decreasing. So we have

$$
\sup _{t \in[0, T]_{\mathbb{T}}}|u(t)|=u(0)
$$

By the concavity of $u(t)$, for $t \in(0, T)_{\mathbb{T}}$, there is

$$
\begin{gathered}
\frac{u(T)-u(0)}{T-0} \geq \frac{u(T)-u(t)}{T-t}, \\
u(0)(T-t) \leq u(t) T-t u(T) \leq u(t) T .
\end{gathered}
$$

Then we have

$$
u(t) \geq \frac{T-t}{T} \sup _{t \in[0, T]_{\mathbb{T}}}|u(t)|
$$

The proof is complete. 
Let $\gamma$ and $\theta$ be nonnegative continuous convex functionals on $P$, let $\alpha$ be a nonnegative continuous concave functional on $P$, and let $\psi$ be a nonnegative continuous functional on $P$. Then for positive real numbers $a, b, c$, and $d$, we define the following convex sets:

$$
\begin{gathered}
P(\gamma, d)=\{x \in P \mid \gamma(x)<d\}, \\
\tilde{P}(\gamma, \alpha, b, d)=\{x \in P \mid b \leq \alpha(x), \gamma(x) \leq d\}, \\
\bar{P}(\gamma, \theta, \alpha, b, c, d)=\{x \in P \mid b \leq \alpha(x), \theta(x) \leq c, \gamma(x) \leq d\},
\end{gathered}
$$

and a closed set

$$
R(\gamma, \psi, a, d)=\{x \in P \mid a \leq \psi(x), \gamma(x) \leq d\} .
$$

The following fixed point theorem due to Avery and Peterson is fundamental in the proof of our main results.

Lemma 2.4 (see [22]). Let $P$ be a cone in a real Banach space E. Let $\gamma$ and $\theta$ be nonnegative continuous convex functionals on $P$, let $\alpha$ be a nonnegative continuous concave functional on $P$, and let $\psi$ be a nonnegative continuous functional on $P$ satisfying $\psi(\lambda x) \leq \lambda \psi(x)$ for $0 \leq \lambda \leq 1$, such that for some positive numbers $\bar{M}$ and $d$,

$$
\alpha(x) \leq \psi(x), \quad\|x\| \leq \bar{M} \gamma(x)
$$

for all $x \in \overline{P(\gamma, d)}$. Suppose that $A: \overline{P(\gamma, d)} \rightarrow \overline{P(\gamma, d)}$ is completely continuous and there exist positive numbers $a, b$, and $c$ with $a<b$ such that

$$
\begin{aligned}
& \left(S_{1}\right)\{x \in \bar{P}(\gamma, \theta, \alpha, b, c, d) \mid \alpha(x)>b\} \neq \emptyset \text { and } \alpha(A x)>b \text { for } x \in \bar{P}(\gamma, \theta, \alpha, b, c, d) ; \\
& \left(S_{2}\right) \alpha(A x)>b \text { for } x \in \widetilde{P}(\gamma, \alpha, b, d) \text { with } \theta(A x)>c ; \\
& \left(S_{3}\right) 0 \notin R(\gamma, \psi, a, d) \text { and } \psi(A x)<a \text { for } x \in R(\gamma, \psi, a, d) \text { with } \psi(x)=a .
\end{aligned}
$$

Then $A$ has at least three fixed points $x_{1}, x_{2}, x_{3} \in \overline{P(\gamma, d)}$, such that

$$
\begin{gathered}
r\left(x_{i}\right) \leq d \quad \text { for } i=1,2,3, \quad b<\alpha\left(x_{1}\right), \\
a<\psi\left(x_{2}\right) \quad \text { with } \alpha\left(x_{2}\right)<b, \quad \psi\left(x_{3}\right)<a .
\end{gathered}
$$

\section{Existence Results}

In this section, by using the Avery-Peterson fixed point theorem, we shall give the sufficient conditions for the existence of at least three positive solutions to the BVPs (1.5) and (1.6). 
Firstly, we define the nonnegative continuous concave functional $\alpha$, the nonnegative continuous convex functionals $\theta, \gamma$, and the nonnegative continuous functional $\psi$ on $P$, respectively, by

$$
\begin{gathered}
r(u)=\sup _{t \in[0, T]_{\mathbb{T}^{k}}}\left|u^{\Delta}(t)\right|=\max \left\{\left|u^{\Delta}(0)\right|,\left|u^{\Delta}(T)\right|\right\}, \\
\psi(u)=\theta(u)=\sup _{t \in[0, T]_{\mathbb{T}}}|u(t)| \leq\|u\|_{1}, \\
\alpha(u)=\inf _{t \in[0, l]_{\mathbb{T}}}|u(t)|=u(l) \quad \text { for } u \in P .
\end{gathered}
$$

For notation convenience, we denote

$$
\begin{gathered}
L=T \phi_{q}\left(\int_{0}^{T} a(\tau) \nabla \tau\right), \quad M=B_{2} \sum_{i=1}^{m-2} b_{i} \int_{0}^{l} \phi_{q}\left(\int_{0}^{s} a(\tau) \nabla \tau\right) \nabla s, \\
N=\left(T^{2}+T B_{1} \sum_{i=1}^{m-2} b_{i}\right) \phi_{q}\left(\int_{0}^{T} a(\tau) \nabla \tau\right), \quad \rho=\frac{2 T}{T-l} .
\end{gathered}
$$

Now we state and prove our main result.

Theorem 3.1. Let $0<a<N b / M \leq \min \{N d(T-l) / 2 M, d N / L\}$ and suppose that $f$ satisfies the following conditions:

$$
\begin{aligned}
& \left(A_{1}\right) f(t, u, v) \leq \phi_{p}(d / L) \text { for }(t, u, v) \in[0, T]_{\mathbb{T}} \times[0, K d] \times[-d, d], \\
& \left(A_{2}\right) f(t, u, v)>\phi_{p}(b / M) \text { for }(t, u, v) \in[0, l]_{\mathbb{T}} \times[b, \rho b] \times[-d, d], \\
& \left(A_{3}\right) f(t, u, v)<\phi_{p}(a / N) \text { for }(t, u, v) \in[0, T]_{\mathbb{T}} \times[0, a] \times[-d, d] .
\end{aligned}
$$

Then problems (1.5) and (1.6) have at least three positive solutions $u_{1}, u_{2}$, and $u_{3}$ such that

$$
\begin{gathered}
\sup _{t \in[0, T]_{\mathbb{T}}}\left|u_{i}^{\Delta}(t)\right| \leq d \quad \text { for } i=1,2,3, \quad b<\inf _{t \in[0, l]_{\mathbb{T}}}\left|u_{1}(t)\right|, \quad \sup _{t \in[0, T]_{\mathbb{T}}}\left|u_{1}(t)\right| \leq K d, \\
a<\sup _{t \in[0, T]_{\mathbb{T}}}\left|u_{2}(t)\right| \quad \text { with } \inf _{t \in[0, l]_{\mathbb{T}}}\left|u_{2}(t)\right|<b, \quad \sup _{t \in[0, T]_{\mathbb{T}}}\left|u_{3}(t)\right|<a .
\end{gathered}
$$

Proof. Define an integral operator $A: P \rightarrow X$ by

$$
\begin{aligned}
(A u)(t)= & \int_{0}^{t}(t-s) \phi_{q}\left(\int_{0}^{s}-a(\tau) f\left(\tau, u(\tau), u^{\Delta}(\tau)\right) \nabla \tau\right) \nabla s \\
& +\int_{0}^{T}(T-s) \phi_{q}\left(\int_{0}^{s} a(\tau) f\left(\tau, u(\tau), u^{\Delta}(\tau)\right) \nabla \tau\right) \nabla s \\
& -B_{0}\left(\sum_{i=1}^{m-2} b_{i} \int_{0}^{\xi_{i}} \phi_{q}\left(\int_{0}^{s}-a(\tau) f\left(\tau, u(\tau), u^{\Delta}(\tau)\right) \nabla \tau\right) \nabla s\right)
\end{aligned}
$$


for $t \in[0, T]_{\mathbb{T}}$. It is easy to obtain that $A: P \rightarrow P$ is a completely continuous operator and every fixed point of $A$ is a solution of (1.5) and (1.6).

Thus we set out to verify that the operator $A$ satisfies Avery-Peterson fixed point theorem which will prove the existence of three fixed points of $A$. Now the proof is divided into some steps.

By virtue of $\alpha(u)=\inf _{t \in[0, l]_{\mathbb{T}}}|u(t)|, \gamma(u)=\sup _{t \in[0, T]_{\mathbb{T}}}\left|u^{\Delta}(t)\right|, \psi(u)=\sup _{t \in[0, T]_{\mathbb{T}}}|u(t)|$ and Lemma 2.2 we know that there exists a constant $\bar{M}$ such that

$$
\alpha(u) \leq \psi(u), \quad\|u\|_{1} \leq \bar{M} \gamma(u) \quad \text { for } u \in \overline{P(\gamma, d)} .
$$

We first show that $\left(A_{1}\right)$ implies that

$$
A: \overline{P(\gamma, d)} \longrightarrow \overline{P(\gamma, d)}
$$

In fact, for $u \in \overline{P(\gamma, d)}, \gamma(u)=\sup _{t \in[0, T]_{\mathbb{T}}}\left|u^{\Delta}(t)\right| \leq d$, by Lemma 2.2, there is $\sup _{t \in[0, T]_{T}}|u(t)| \leq K d$. It follows from $\left(A_{1}\right)$ that

$$
\begin{aligned}
\gamma(A(u)) & =\sup _{t \in[0, T]_{\mathbb{T}}}\left|(A u)^{\Delta}(t)\right|=\sup _{t \in[0, T]_{\mathbb{T}}}\left|\int_{0}^{t} \phi_{q}\left(\int_{0}^{s} a(\tau) f\left(\tau, u(\tau), u^{\Delta}(\tau)\right) \nabla \tau\right) \nabla s\right| \\
& \leq \int_{0}^{T} \phi_{q}\left(\int_{0}^{T} a(\tau) f\left(\tau, u(\tau), u^{\Delta}(\tau)\right) \nabla \tau\right) \nabla s \\
& \leq \frac{T d}{L} \phi_{q}\left(\int_{0}^{T} a(\tau) \nabla \tau\right)=d .
\end{aligned}
$$

Thus (3.6) holds.

Next we show that condition $\left(S_{1}\right)$ in Lemma 2.4 holds. Let $\bar{u}(t)=2 b(T-t) /(T-l)$. Then it is easy to see that $\bar{u}(t) \geq 0, \bar{u}(T)+B_{0}\left(\sum_{i=1}^{m-2} b_{i} \bar{u}^{\Delta}\left(\xi_{i}\right)\right) \leq 0, \bar{u}^{\Delta}(0) \leq 0$, and $\bar{u}^{\Delta \nabla}(t) \leq 0$ for $t \in[0, T]_{\mathbb{T}}$, so $\bar{u} \in P$. Also, we have

$$
\begin{aligned}
& r(\bar{u})=\sup _{t \in[0, T]_{\mathbb{T}^{k}}}\left|u^{\Delta}(t)\right|=\frac{2 b}{T-l} \leq d, \\
& \theta(\bar{u})=\sup _{t \in[0, T]_{\mathbb{T}}}|u(t)|=\frac{2 b T}{T-l} \leq \rho b, \\
& \alpha(\bar{u})=\inf _{t \in[0, l]_{\mathbb{T}}}|u(t)|=2 b>b .
\end{aligned}
$$

So $\bar{u} \in \bar{P}(\gamma, \theta, \alpha, b, \rho b, d)$. Hence $\{u \in \bar{P}(\gamma, \theta, \alpha, b, \rho b, d) \mid \alpha(u)>b\} \neq \emptyset$. 
If $u \in \bar{P}(\gamma, \theta, \alpha, b, \rho b, d)$, then $b \leq u(t) \leq \rho b,\left|u^{\Delta}(t)\right| \leq d$ for $0 \leq t \leq l$. It follows from condition $\left(A_{2}\right)$ that

$$
\begin{aligned}
\alpha(A u)= & \inf _{t \in[0,]_{\mathbb{T}}}|(A u)(t)|=(A u)(l) \\
= & \int_{0}^{l}(l-s) \phi_{q}\left(\int_{0}^{s}-a(\tau) f\left(\tau, u(\tau), u^{\Delta}(\tau)\right) \nabla \tau\right) \nabla s \\
& +\int_{0}^{T}(T-s) \phi_{q}\left(\int_{0}^{s} a(\tau) f\left(\tau, u(\tau), u^{\Delta}(\tau)\right) \nabla \tau\right) \nabla s \\
& -B_{0}\left(\sum_{i=1}^{m-2} b_{i} \int_{0}^{\xi_{i}} \phi_{q}\left(\int_{0}^{s}-a(\tau) f\left(\tau, u(\tau), u^{\Delta}(\tau)\right) \nabla \tau\right) \nabla s\right) \\
\geq & -B_{0}\left(\sum_{i=1}^{m-2} b_{i} \int_{0}^{\xi_{i}} \phi_{q}\left(\int_{0}^{s}-a(\tau) f\left(\tau, u(\tau), u^{\Delta}(\tau)\right) \nabla \tau\right) \nabla s\right) \\
\geq & B_{2} \sum_{i=1}^{m-2} b_{i} \int_{0}^{l} \phi_{q}\left(\int_{0}^{s} a(\tau) f\left(\tau, u(\tau), u^{\Delta}(\tau)\right) \nabla \tau\right) \nabla s \\
> & \frac{b}{M} B_{2} \sum_{i=1}^{m-2} b_{i} \int_{0}^{l} \phi_{q}\left(\int_{0}^{s} a(\tau) \nabla \tau\right) \nabla s=b .
\end{aligned}
$$

Therefore we have

$$
\alpha(A u)>b \text { for } u \in \bar{P}(\gamma, \theta, \alpha, b, \rho b, d) .
$$

That is, condition $\left(S_{1}\right)$ in Lemma 2.4 is satisfied.

We now prove that $\left(S_{2}\right)$ in Lemma 2.4 holds. In fact, since $\alpha(A u)=A u(l), \theta(A u)=$ $\sup _{t \in[0, T]_{\mathbb{T}}}|A u(t)|$, then with Lemma 2.3 it follows that

$$
\alpha(A u) \geq \frac{T-l}{T} \theta(A u)>\frac{T-l}{T} \rho b \geq b
$$

for $u \in \widetilde{P}(\gamma, \alpha, b, d)$ with $\theta(A u)>\rho b$. Hence condition $\left(S_{2}\right)$ in Lemma 2.4 is satisfied.

Finally, we assert that $\left(S_{3}\right)$ in Lemma 2.4 also holds. 
Observe that $\psi(0)=0<a$, so $0 \notin R(\gamma, \psi, a, d)$. Suppose $u \in R(\gamma, \psi, a, d)$ with $\psi(u)=a$. Then, by hypothesis $\left(A_{3}\right)$ we have

$$
\begin{aligned}
\psi(A(u))= & \sup _{t \in[0, T]_{\mathbb{T}}}|(A u)(t)|=(A u)(0) \\
= & \int_{0}^{T}(T-s) \phi_{q}\left(\int_{0}^{s} a(\tau) f\left(\tau, u(\tau), u^{\Delta}(\tau)\right) \nabla \tau\right) \nabla s \\
& -B_{0}\left(\sum_{i=1}^{m-2} b_{i} \int_{0}^{\xi_{i}} \phi_{q}\left(\int_{0}^{s}-a(\tau) f\left(\tau, u(\tau), u^{\Delta}(\tau)\right) \nabla \tau\right) \nabla s\right) \\
< & \int_{0}^{T} T \phi_{q}\left(\int_{0}^{s} a(\tau) f\left(\tau, u(\tau), u^{\Delta}(\tau)\right) \nabla \tau\right) \nabla s \\
& +B_{1} \sum_{i=1}^{m-2} b_{i} \int_{0}^{T} \phi_{q}\left(\int_{0}^{s} a(\tau) f\left(\tau, u(\tau), u^{\Delta}(\tau)\right) \nabla \tau\right) \nabla s \\
\leq & \left.\frac{T^{2} a}{N} \phi_{q}\left(\int_{0}^{T} a(\tau) \nabla \tau\right)+\frac{T B_{1} a^{m-2} \sum_{i=1}^{m} b_{i} \phi_{q}\left(\int_{0}^{T} a(\tau) \nabla \tau\right)}{N}\right) \\
= & \left(T^{2}+T B_{1} \sum_{i=1}^{m-2} b_{i}\right) \frac{a}{N} \phi_{q}\left(\int_{0}^{T} a(\tau) \nabla \tau\right)=a .
\end{aligned}
$$

Thus condition $\left(S_{3}\right)$ in Lemma 2.4 holds.

Therefore an application of Lemma 2.4 implies that the BVPs (1.5) and (1.6) have at least three positive solutions $u_{1}, u_{2}$, and $u_{3}$ such that (3.3) holds.

\section{Example}

In this section, we present an example to explain our result.

Let $\mathbb{T}=\{0\} \bigcup\left\{1 / 2^{n}, n \in \mathbb{N}_{0}\right\}, a(t) \equiv 1, p=3$, and $m=4, \xi_{1}=1 / 4, \xi_{2}=1 / 2, b_{1}=b_{2}=1$, $B_{0}(x)=2 x$. We consider the following boundary value problem:

$$
\begin{gathered}
\left(\left|u^{\Delta \nabla}(t)\right| u^{\Delta \nabla}(t)\right)^{\nabla}+f\left(t, u(t), u^{\Delta}(t)\right)=0, \quad t \in[0,1]_{\mathbb{T}} \\
u^{\Delta}(0)=u^{\Delta \nabla}(0)=0, \quad u(1)+2\left(u^{\Delta} \frac{1}{4}+u^{\Delta} \frac{1}{2}\right)=0
\end{gathered}
$$


where

$$
f(t, u, v)= \begin{cases}\frac{1}{10^{3}} t+\frac{1}{5} \times u^{12}+\frac{1}{7}\left(\frac{v}{2.4 \times 10^{7}}\right)^{2}, & u \leq 2 \\ \frac{1}{10^{3}} t+\frac{1}{5} \times 2^{12}+\frac{1}{7}\left(\frac{v}{2.4 \times 10^{7}}\right)^{2}, & u>2 .\end{cases}
$$

Choosing $a=1 / 2, b=2, l=1 / 4, d=2.4 \times 10^{6}$, direct calculation shows that

$$
\rho=\frac{8}{3}, \quad K=5, \quad L=1, \quad M=\frac{4+\sqrt{2}}{56}, \quad N=5 .
$$

Consequently, $f(t, u, v)$ satisfies

(i) $f(t, u, v)<822<\phi_{3}(d / L)=5.76 \times 10^{12}$ for $(t, u, v) \in[0,1]_{\mathbb{T}} \times\left[0,1.2 \times 10^{7}\right] \times[-2.4 \times$ $\left.10^{6}, 2.4 \times 10^{6}\right]$;

(ii) $f(t, u, v)=4096 / 5>\phi_{3}(b / M)=128(9-4 \sqrt{2})$ for $(t, u, v) \in[0,1 / 4]_{\mathbb{T}} \times[2,16 / 3] \times$ $\left[-2.4 \times 10^{6}, 2.4 \times 10^{6}\right]$;

(iii) $f(t, u, v) \leq 4 / 1000<\phi_{3}(a / N)=1 / 100$ for $(t, u, v) \in[0,1]_{\mathbb{T}} \times[0,1 / 2] \times[-2.4 \times$ $\left.10^{6}, 2.4 \times 10^{6}\right]$.

Then all conditions of Theorem 3.1 hold. Thus with Theorem 3.1, the BVP (4.1) has at least three positive solutions $u_{1}, u_{2}$, and $u_{3}$ such that

$$
\begin{gathered}
\sup _{t \in[0,1]_{\mathbb{T}^{\kappa}}}\left|u_{i}^{\Delta}(t)\right| \leq 2.4 \times 10^{6} \quad \text { for } i=1,2,3, \quad 2<\inf _{t \in[0,1 / 4]_{\mathbb{T}}}\left|u_{1}(t)\right|, \quad \sup _{t \in[0,1]_{\mathbb{T}}}\left|u_{1}(t)\right| \leq 1.2 \times 10^{7}, \\
\frac{1}{2}<\sup _{t \in[0,1]_{\mathbb{T}}}\left|u_{2}(t)\right| \quad \text { with } \inf _{t \in[0,1 / 4]_{\mathbb{T}}}\left|u_{2}(t)\right|<2, \quad \sup _{t \in[0,1]_{\mathbb{T}}}\left|u_{3}(t)\right|<\frac{1}{2} .
\end{gathered}
$$

\section{Acknowledgment}

Supported by the NNSF of China (10801065) and NSF of Gansu Province of China (0803RJZA096).

\section{References}

[1] S. Hilger, Ein Maßkettenkalkül mit Anwendung auf Zentrumsmannigfaltigkeiten, Ph.D. thesis, Universität Würzburg, Würzburg, Germany, 1988.

[2] V. Spedding, “Taming nature's numbers," New Scientist, pp. 28-32, July 2003.

[3] D. M. Thomas, L. Vandemuelebroeke, and K. Yamaguchi, "A mathematical evolution model for phytoremediation of metals," Discrete and Continuous Dynamical Systems B, vol. 5, no. 2, pp. 411-422, 2005.

[4] R. P. Agarwal, V. O. Espinar, K. Perera, and D. R. Vivero, "Multiple positive solutions in the sense of distributions of singular BVPs on time scales and an application to Emden-Fowler equations," Advances in Difference Equations, vol. 2008, Article ID 796851, 13 pages, 2008. 
[5] R. P. Agarwal, V. O. Espinar, K. Perera, and D. R. Vivero, "Multiple positive solutions of singular Dirichlet problems on time scales via variational methods," Nonlinear Analysis: Theory, Methods $\mathcal{E}$ Applications, vol. 67, no. 2, pp. 368-381, 2007.

[6] M. Bohner and A. Peterson, Dynamic Equations on Time Scales, Birkhäuser, Boston, Mass, USA, 2001.

[7] M. Bohner and A. Peterson, Advances in Dynamic Equations on Time Scales, Birkhäuser, Boston, Mass, USA, 2003.

[8] W. T. Li and H. R. Sun, "Positive solutions for second-order $p$-point boundary value problems on time scales," Acta Mathematica Sinica, vol. 22, no. 6, pp. 1797-1804, 2006.

[9] Y. Wang and W. Ge, "Positive solutions for multipoint boundary value problems with a onedimensional p-Laplacian," Nonlinear Analysis: Theory, Methods \& Applications, vol. 66, no. 6, pp. 1246 1256, 2007.

[10] B. Zhao and H. R. Sun, "Multiplicity results of positive solution for nonlinear three-point boundary value problem on time scales," Advances in Dynamical Systems and Applications, vol. 4, no. 2, pp. 25-43, 2009.

[11] J. Henderson, "Multiple solutions for $2 m$-th order Sturm-Liouville boundary value problems on a measure chain," Journal of Difference Equations and Applications, vol. 6, no. 4, pp. 417-429, 2000.

[12] S. H. Li, "Positive solutions of nonlinear singular third-order two-point boundary value problem," Journal of Mathematical Analysis and Applications, vol. 323, no. 1, pp. 413-425, 2006.

[13] Y. P. Sun, "Positive solutions of singular third-order three-point boundary value problem," Journal of Mathematical Analysis and Applications, vol. 306, no. 2, pp. 589-603, 2005.

[14] İ. Yaslan, "Existence results for an even-order boundary value problem on time scales," Nonlinear Analysis: Theory, Methods \& Applications, vol. 70, no. 1, pp. 483-491, 2009.

[15] Y.-H. Su, W.-T. Li, and H.-R. Sun, "Positive solutions of singular $p$-Laplacian BVPs with sign changing nonlinearity on time scales," Mathematical and Computer Modelling, vol. 48, no. 5-6, pp. 845-858, 2008.

[16] H.-R. Sun and W.-T. Li, "Existence theory for positive solutions to one-dimensional $p$-Laplacian boundary value problems on time scales," Journal of Differential Equations, vol. 240, no. 2, pp. 217248, 2007.

[17] C. L. Zhou and D. X. Ma, "Existence and iteration of positive solutions for a generalized right-focal boundary value problem with $p$-Laplacian operator," Journal of Mathematical Analysis and Applications, vol. 324, no. 1, pp. 409-424, 2006.

[18] D. R. Anderson and A. Cabada, "Third-order right-focal multi-point problems on time scales," Journal of Difference Equations and Applications, vol. 12, no. 9, pp. 919-935, 2006.

[19] W. Han and S. Kang, "Multiple positive solutions of nonlinear third-order BVP for a class of $p$ Laplacian dynamic equations on time scales," Mathematical and Computer Modelling, vol. 49, no. 3-4, pp. 527-535, 2009.

[20] K. Deimling, Nonlinear Functional Analysis, Springer, Berlin, Germany, 1985.

[21] D. J. Guo and V. Lakshmikantham, Nonlinear Problems in Abstract Cones, vol. 5 of Notes and Reports in Mathematics in Science and Engineering, Academic Press, Boston, Mass, USA, 1988.

[22] R. I. Avery and A. C. Peterson, "Three positive fixed points of nonlinear operators on ordered Banach spaces," Computers \& Mathematics with Applications, vol. 42, no. 3-5, pp. 313-322, 2001. 